\title{
EXERCISE AND HEART DISEASE
}

\author{
Alfred A. Bove, M.D., Ph.D. \\ Temple University School of Medicine, Philadelphia, Pennsylvania
}

Lifestyle management plays an important role in the world of cardiovascular care, and for most cardiovascular diseases, exercise is listed as an essential component of lifestyle advice. The recent guidelines from the American College of Cardiology and American Heart Association include an entire section on how to use lifestyle information to optimally manage patients with or at risk for cardiovascular disease. ${ }^{1}$ Many of our patients are older adults, and many have heart failure with preserved or reduced ejection fraction. We may be consulted by middle aged or younger individuals who want to compete in sports events. Distance runs and triathlons are becoming more popular, and many adults consult a cardiologist before participating in competitive sports. Since cardiologists will likely need to provide exercise advice to nearly all of our patients, it is important to understand the circulatory and pulmonary physiology involved in exercise.

The paper on pulmonary aspects of exercise points out several important concepts that can be applied to all patients. Data are now clear that exercise improves left ventricular compliance, and a highly compliant ventricle that can relax rapidly is essential to minimizing dyspnea with exercise. The fact that exercise can be used as a therapeutic measure in heart failure with preserved ejection fraction provides a tool to help manage patients with this form of cardiovascular disease. The lung responses to exercise follow the hemodynamic changes. Cardiologists are seeing athletes with swimming-induced pulmonary edema, and a discussion of the disorder is provided in the first review. Similarly, a resurgence of the sport of deep breath hold diving supports the need for an understanding of the dynamics of this activity, which also is provided in the first paper. Indeed, recent data show that even normal athletes exercising to extremes can develop pulmonary edema in the background of normal lungs. Many of these patients appear in the cardiologist office for evaluation of cardiac status, and most do not show significant cardiac pathology.

The paper by Zaleski et al. provides a detailed background on how to inform older adults ( $>65$ years old) about the value of exercise. This group of patients constitutes many in our daily practice, and while we focus on managing comorbidities such as hypertension, hyperlipidemia, chronic stable angina, and valvular heart disease, lifestyle advice remains one of the key additions that we should provide for this population. Zaleski and colleagues point out that the expanding older population must manage a number of comorbidities as they age, and they offer specific recommendations so that the cardiologist can provide advice about exercise based on well-established guidelines. Of importance in this group is the need for resistance exercise to aide in maintaining bone health, balance, and general conditioning. The first consideration for cardiologists who advise older individuals about exercise programs is safety. Since many older patients have some degree of coronary atherosclerosis, the risk of a coronary event should be the primary consideration when providing exercise advice. This risk can be stratified with a medical history and an organized process of health screening. The screening process is described in detail in this paper and can be used to manage the older adult who embarks on an exercise program.

The series then moves from the healthy adult to the adult patient with heart failure-a population that is projected to exceed 8.5 million by $2030 .^{2}$ As with other cardiovascular disorders, lifestyle is an important component of care. Although the use of exercise therapy in heart failure patients has been supported by several clinical trials, the most recent being the HF-ACTION study, ${ }^{3}$ Alvarez and colleagues point out that only $10.4 \%$ of eligible heart failure patients receive cardiac rehabilitation after hospitalization. They go on to provide several methods for assessing exercise capacity in these patients and the limitations imposed by left ventricular dysfunction. They also review the benefits of exercise in VAD and transplant patients. For centers that manage patients with advanced heart failure, most have the capacity for formal cardiopulmonary exercise testing that provides a measure of actual maximum oxygen consumption. While this is the best way to evaluate exercise capacity in heart failure patients, a commonly used alternative is the 6-minute walk test that measures how far a heart failure patient can walk in 6 minutes. This is something that can easily be done in an office or hospital setting, without a formal testing area.

There is a growing population of patients who have heart failure with preserved ejection fraction (HFpEF). This is due to the increasing lifespan of our cardiac patients, the incidence of hypertension and left ventricular (LV) hypertrophy in the population, and the presence of coronary disease, either overt or occult. While HFpEF has become a dominant cause of clinical heart failure in the older population, most clinical trials have failed to show an advantage for our usual heart failure medications. Gupte and Hamilton provide an excellent overview of this growing problem for the clinical cardiologist. Their review of the pathophysiology highlights the problem of elevated end diastolic pressure, failure of LV relaxation, and pulmonary venous pressure that leads first to dyspnea and then to overt pulmonary edema. The mechanisms are a subset of similar mechanisms that occur in normal hearts and in athletes who extend their exercise capacity beyond limits of normal pulmonary capillary pressure. Recent studies demonstrate that exercise training can reduce diastolic stiffness and improve exercise capacity. Even so, HFpEF is a growing challenge in cardiology practice since the mechanisms causing this disorder are not well understood, and both basic and clinical research is needed to improve our care of these patients.

Next, Wasfy and colleagues take us in a different direction, pointing out the concern for sudden cardiac death (SCD) in athletes. This has become a timely topic that involves several issues, including screening, selection of athletes with heart disease for sport participation, and the continuing question of how to detect the at-risk athlete before an event occurs. For the adult athlete over age 35, the most common cause of SCD is coronary disease; therefore, this population needs evaluation and screening for SCD risk based on well-defined risk factors 
such as hypertension, hyperlipidemia, diabetes, and family history of premature coronary disease. Guideline-directed screening, including stress testing, should be considered in this population. For younger athletes, where etiology is often an inherited disorder (e.g., hypertrophic cardiomyopathy, dilated cardiomyopathy, channelopathies, Marfan syndrome), the authors highlight the American Heart Association's 12-point history and physical examination, which is the current standard for athletic participation. ${ }^{4}$ For these athletes, electrocardiographic (ECG) screening remains controversial. The authors emphasize that athletic screenings should be done by cardiologists familiar with the physiologic changes that occur in the heart with physical training. Without this in-depth knowledge, many athletes will be stigmatized with a cardiac diagnosis based on ECG or imaging data that reflects normal cardiac adaptations to physical training. This advice follows several years of controversy about ECG screening of athletes and the continuing concern for high false-positive rates, particularly when interpretation is based on usual ECG criteria and the reader is unfamiliar with expected ECG changes in a trained athlete.

The paper by Lisman provides the details needed to understand adaptive cardiac changes in athletes. He discusses how specific types of training can produce cardiac changes that may appear abnormal on the ECG and consequently lead to high false-positive rates that exclude healthy individuals. By using the Seattle criteria to interpret the ECG in athletes, Lisman shows how cardiologists can differentiate between normal versus abnormal cardiac changes that may signal an underlying condition and how these criteria have reduced the number of false-positive readings.

For the cardiologist involved in screening athletes of any age, much of the information provided here will be invaluable for helping patients who wish to start an exercise program, compete in sports events, use exercise as a therapeutic tool for improving heart failure symptoms, or reduce cardiovascular risk as part of lifestyle goals for prevention. Further information on cardiology practice trends can be found in the 2016 AHA Heart and Stroke Statistics. ${ }^{5}$

\section{References}

1. Eckel RH, Jakicic JM, Jamy D, et al. 2013 AHA/ACC Guideline on Lifestyle Management to Reduce Cardiovascular Risk. A Report of the American College of Cardiology/American Heart Association Task Force on Practice Guidelines. J Am Coll Cardiol. 2014 Jul;63(25):2960-84.

2. Heidenreich PA, Trogdon JG, Khavjou OA, et al. Forecasting the future of cardiovascular disease in the United States: a policy statement from the American Heart Association. Circulation. 2011;123:933-44.

3. O'Connor CM, Whellan DJ, Lee KL, et al.; HF-ACTION Investigators. Efficacy and safety of exercise training in patients with chronic heart failure: HF-ACTION randomized controlled trial. JAMA. 2009 Apr 8;301(14):1439-50.

4. Maron BJ, Thompson PD, Ackerman MJ, et al.; American Heart Association Council on Nutrition, Physical Activity, and Metabolism. Recommendations and considerations related to preparticipation screening for cardiovascular abnormalities in competitive athletes: 2007 update: a scientific statement from the American Heart Association Council on Nutrition, Physical Activity, and Metabolism: endorsed by the American College of Cardiology Foundation. Circulation. 2007 Mar 27;115(12):1643-455.

5. Mozaffarian D, Benjamin EJ, Go AS, et al; American Heart Association Statistics Committee and Stroke Statistics Subcommittee. Heart Disease and Stroke Statistics-2016 Update: A Report From the American Heart Association. Circulation. 2016 Jan 26;133(4):e38-e360. 